Abanico Veterinario. Enero-Diciembre 2021; 11:1-7. http://dx.doi.org/10.21929/abavet2021.28 Caso Clínico. Recibido: 26/10/2020. Aceptado: 03/06/2021. Publicado: 10/06/2021. Clave: e2020-87.

\title{
Laminitis en una yegua Pura Raza Española en Tabasco, México: Reporte de caso
}

\author{
Laminitis in a purebred Spanish mare in Tabasco, Mexico: Case report
}

\author{
Frías-de-la-Cruz José ${ }^{\star 11 D}$, García-Herrera Ricardo ${ }^{11 D}$, Portillo-Salgado Rodrigo ${ }^{21 D}$, \\ Cigarroa-Vázquez Francisco ${ }^{31 \mathrm{D}}$, Chay-Canul Alfonso**1ID, Herrera-Camacho José 4 ID
}

1'División Académica de Ciencias Agropecuarias, Universidad Juárez Autónoma de Tabasco. ${ }^{2}$ Programa de Ganadería, Colegio de Postgraduados, Campus Montecillo. ${ }^{3}$ Universidad Autónoma de Chiapas, Facultad de Medicina Veterinaria y Zootecnia. ${ }^{4}$ Instituto de Investigaciones Agropecuarias y Forestales, Universidad Michoacana de San Nicolás de Hidalgo. "Autor responsable: Frías de la Cruz José E. "Autor de correspondencia: Chay-Canul Alfonso J. División Académica de Ciencias Agropecuarias, Universidad Juárez Autónoma de Tabasco. Carretera Villahermosa-Teapa, km 25, CP 86280. Villahermosa, Tabasco, México. E-mail: josefriasmvz@gmail.com, ricardogarciaherrera@hotmail.com, rps_1303@hotmail.com, antonio.cigarroa@unach.mx, aljuch@hotmail.com, jose.camacho@umich.mx

\section{RESUMEN}

La laminitis es una enfermedad grave, de alta prevalencia, reconocida como uno de los síndromes clínicos más importantes en equinos. En el presente estudio se describe el caso de una yegua Pura Raza Española de cinco años de edad, con diagnóstico de laminitis bilateral de aproximadamente tres meses. El animal se reusaba a caminar, manteniéndose echado todo el tiempo. El grado de claudicación, según la escala de Obel, se calificó de 5; asimismo, presentaba pulso palmar digital alterado. Se receto terapia de 30 días con antinflamatorios $1.1 \mathrm{mg} / \mathrm{kg}$ intra venosa (IV) de flunixin de meglumine cada $24 \mathrm{~h}$, acompañado de omeprazol $2 \mathrm{mg} / \mathrm{kg}$ vía oral (VO). Adicionalmente, se recomendó recorte de casco y herrajes correctivos. Después de los ocho meses de tratamiento, la yegua presentó una notable mejoría y ganancia de condición corporal, además, se logró una gestación. El tratamiento antiinflamatorio, recorte de casco y herrajes correctivos, favorecieron el crecimiento y dureza del casco, teniendo éxito en el tratamiento de laminitis.

Palabras clave: herraje correctivo, laminitis, Raza Pura Española.

\begin{abstract}
Laminitis is a serious, highly prevalent disease, recognized as one of the most important clinical syndromes in equines. The present study describes the case of a five-year-old Pura Raza Española mare diagnosed with bilateral laminitis at approximately three months of age. The animal refused to walk, lying down all the time. The degree of claudication, according to the Obel scale, was graded as 5; she also had an altered palmar digital pulse. He was prescribed 30 days of anti-inflammatory therapy with $1.1 \mathrm{mg} / \mathrm{kg}$ intravenous (IV) of meglumine flunixin every $24 \mathrm{~h}$, accompanied by omeprazole $2 \mathrm{mg} / \mathrm{kg}$ orally (O.V.). Additionally, hoof trimming and corrective shoeing were recommended. After eight months of treatment, the mare showed a remarkable improvement and gain in body condition, and a gestation was achieved. The anti-inflammatory treatment, hoof trimming and corrective shoeing favored the growth and hardness of the hoof, being successful in the treatment of laminitis.
\end{abstract}

Keywords: corrective shoeing, laminitis, Purebred Spanish Horse.

\section{INTRODUCCIÓN}

La laminitis o infosura es una enfermedad grave, de alta prevalencia, reconocida como uno de los síndromes clínicos más importantes en equinos (Paula et al., 2020). El término laminitis es utilizado para describir una patología sistémica, que compromete la condición general del animal. Es una inflamación de las láminas sensibles del casco que causa degeneración, separación y necrosis del corion laminar (Londoño-Sossa et al., 2011; 
Mitchell et al., 2015). La laminitis provoca un dolor agudo a los animales y es potencialmente mortal, afectando principalmente a los caballos y ponis adultos de cualquier raza (Mitchell et al., 2015).

La laminitis se asocia comúnmente con la ingestión de carbohidratos no estructurales de los pastos; sin embargo, no solo está relacionada con la dieta, sino también con algunas enfermedades sistémicas (Londoño-Sossa et al., 2011; Mitchell et al., 2015; Paula et al., 2020). Las alteraciones clínicas que predisponen la laminitis son: síndrome abdominal agudo, enteritis, retención de las membranas fetales, metritis, pleuroneumonía, y otras patologías. Asimismo, se puede presentar por un manejo inadecuado del animal, como ingestión excesiva de agua fría luego de trabajar o la administración de altos niveles de corticoides, los cuales disminuyen la síntesis de proteínas, potencian la vasoconstricción digital e inducen micro-trombosis (Londoño-Sossa et al., 2011; Mitchell et al., 2015; Paula et al., 2020). Otros factores de riesgo asociados a la laminitis son, la raza del caballo siendo más propensas las raza de tiro, el sobrepeso en el animal, plano nutricional alto y caballos mayores con enfermedad de Cushing (AAEP, 2020).

La laminitis, puede presentarse en una o más extremidades con signos característicos, como cojera, incremento de la temperatura del casco y pulso digital aumentado en la extremidad, dolor cuando se aplica presión, marcha vacilante, postura de caballete con patas delanteras extendidas hacia adelante para aliviar la presión, en casos crónicos; se puede observar ensanchamiento de los anillos de la pared del casco medida que se siguen de la punta al talón, suelas magulladas, línea blanca ensanchada, con aparición de seromas y/o abscesos, suelas caídas, callo grueso así como cascos cóncavos con apariencia de "zapatilla de Aladino" (AAEP, 2020).

La Americans Association of Equine Practitioners (AAEP, 2020) establecio la siguiente escala de cojera en equinos:

Grado 1: La cojera difícil de observar en cualquier situación

Grado 2: La cojera difícil de observar al paso o al trote pero que aparece en

determinadas circunstancias

Grado 3: Cojera permanente en el trote en todo momento

Grado 4: La cojera es evidente asintiendo con la cabeza o dando una zancada corta Grado 5: La cojera es muy evidente y permanente con una carga de peso mínimo y la manifiesta en descanso y mas aun en movimiento

El conocimiento y la comprensión de la fisiopatología de la laminitis son un tanto desconocidos, situación que limita los esfuerzos para su prevención y tratamiento. Por lo tanto, el diagnóstico oportuno y su posterior tratamiento médico, apoyado de métodos biomecánicos, son de suma importancia para minimizar los efectos de esta enfermedad (Mitchell et al., 2015).

El objetivo de este estudio es describir el diagnóstico y tratamiento de un caso de laminitis bilateral en una yegua Pura Raza Española de cinco años de edad, que se trató con terapia antiinflamatoria, recorte de casco y herrajes correctivos. 


\section{DESCRIPCIÓN DEL CASO CLÍNICO}

Se presenta el caso de una yegua Pura Raza Española de cinco años de edad (figura 1), que comenzó con incomodidad al caminar y se mantenía echada la mayor parte del día. Este problema llevaba cerca de tres meses. El grado de claudicación, considerando la escala de la EEAP (2020), se calificó de grado 5; asimismo, presentaba pulso palmar digital aumentado. La yegua permaneció en descanso en una caballeriza con piso de caucho, y con cama de viruta la cual tenía un grosor de aproximadamente $70 \mathrm{~cm}$ para que estuviera lo más cómoda posible. En cuanto a su alimentación, estaba basada en zacate fresco Taiwán (Pennisetum purpureum) picado a libre acceso sin concentrado adicional.

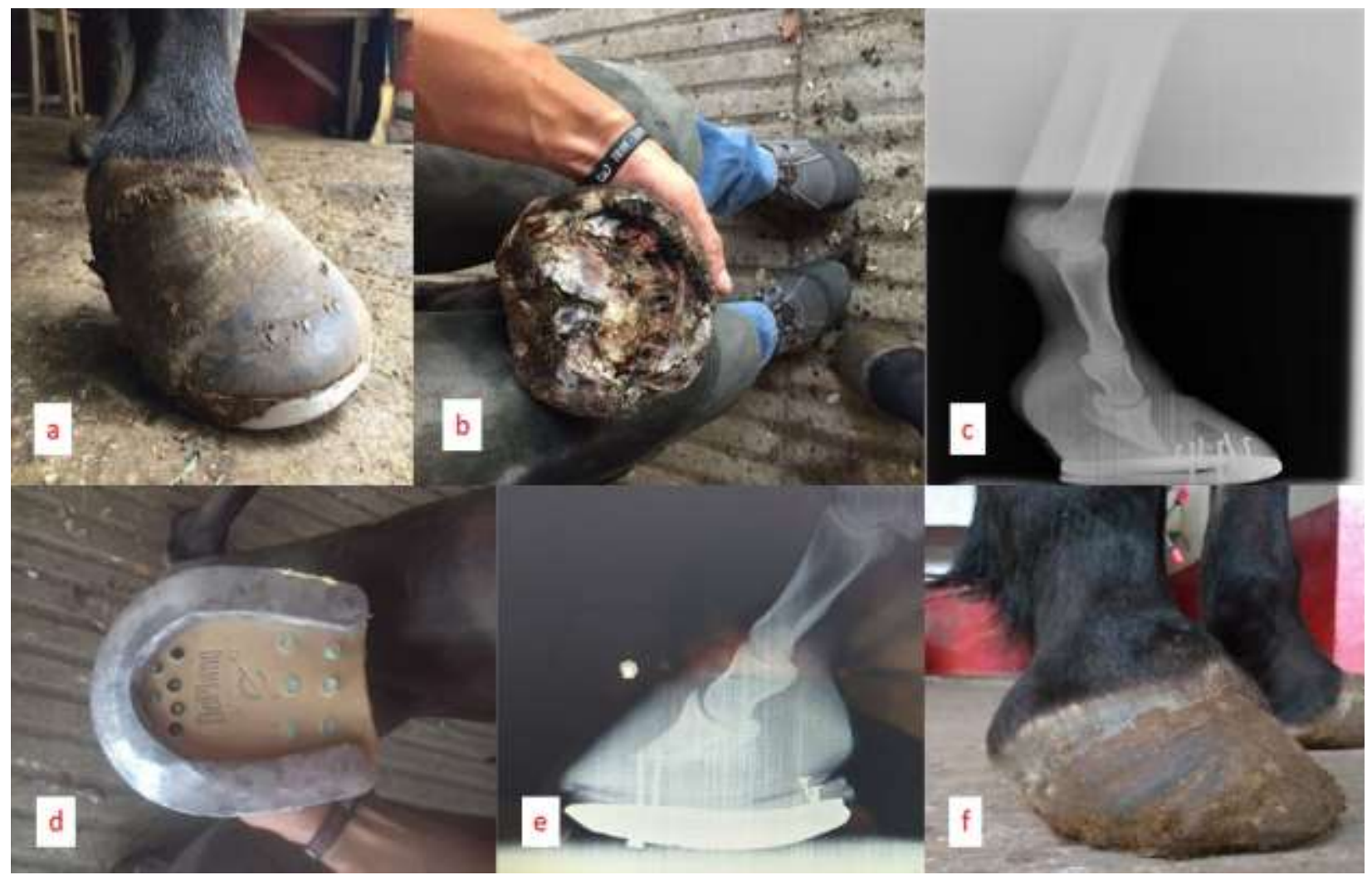

Figura 1. Proceso clínico de laminitis en yegua de la Raza Española. a) Aspecto del casco laminitico, b) A través del examen físico del casco se puede observar claramente la necrosis de las láminas dérmicas y la muerte celular del corion solar y corion de la muralla, c) Imagen latero-medial MAD con evidente rotación de $5^{\circ}$ a punto de perforar la parte solar, d) herraje terapéutico del casco a base de una herradura de aluminio pegada y material de impresión para dar soporte a la parte palmar del casco, e) Aspecto radiográfico del MAl después de 12 meses de tratamiento y f) Aspecto del casco del MAD después 12 meses de tratamiento.

Primeramente, se realizó un examen físico del casco (figura 1a,b) de la extremidad anterior derecha/izquerda, utilizando unas pinzas de palpación. Posteriormente se tomaron radiografías utilizando una proyección latero-medial para conocer el grado de rotación de ambos miembros (figura 1c). Una vez obtenidas las radiografías se estableció 
que el ángulo de rotación de la tercera falange (p3) era superior a $5^{\circ}$. Se realizó el recorte para estabilizar y mejorar la posición de P3 dentro del estuche corneo, de manera que el herraje terapeútico realizará su función de recortar el paso. El herraje utilizado en el tratamiento fue modelo libero (Mustad, Noruega) con modificación tipo rokert toe (figura 1e).

Se receto terapia de 30 días con antinflamatorios $1.1 \mathrm{mg} / \mathrm{kg}$ intravenoso de flunixin de meglumine cada $24 \mathrm{~h}$, acompañado de omeprazol $2 \mathrm{mg} / \mathrm{kg}$ via oral para prevenir posibles ulceras gástricas y prolongar el uso de los antinflamatorios.

Después de 12 meses de tratamiento mediante herraje terapéutico (figura 1f), la yegua presentó una considerable mejoría y ganancia de condición corporal, logrando una gestación.

En la figura 2, se aprecia las imágenes radiográficas de la laminitis al inicio asi como su evolución a los seis y 12 meses después del tratamiento.
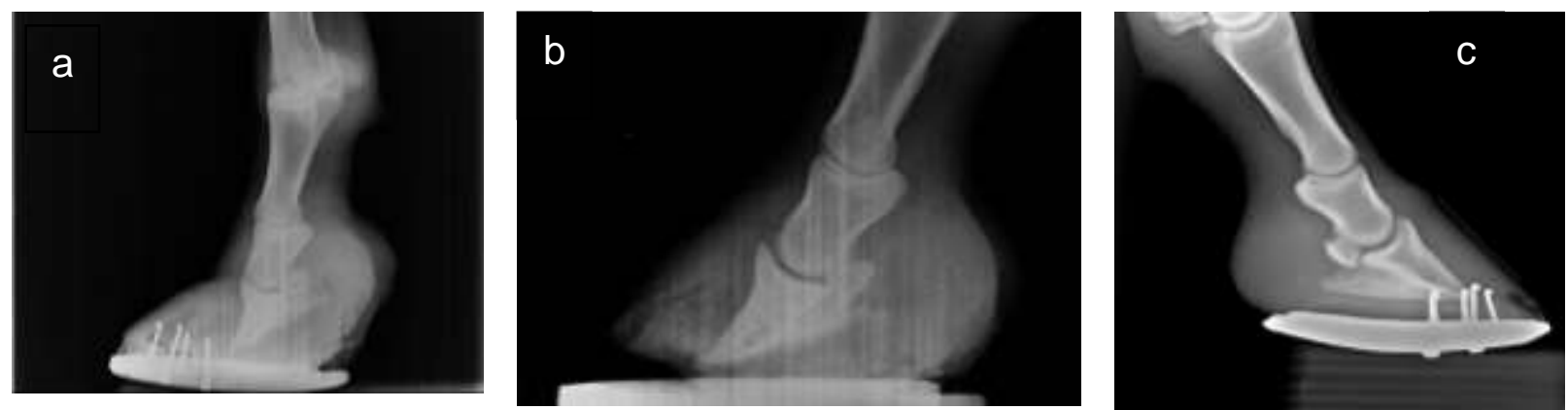

Figura 2. Imágenes radiográficas de laminitis bilateral, a) MAI con rotación de $5^{\circ}$ a punto de perforar la suela; b) MAl a los seis 6 meses posteriores a la desrotacion y tenotomía del tendón flexor común y c) MAD después de 12 meses de tratamiento.

\section{DISCUSIÓN}

El pronóstico oportuno dado por profesionales clínicos éticos es muy importante en el tratamiento de padecimientos en equinos como la laminitis. Los parámetros más recomendados para estimar el pronóstico en los equinos con laminitis son: el grado de claudicación y la intensidad de la rotación de la tercera falange medida como el ángulo que forma el aspecto dorsal del hueso podal y la pared del casco (Londoño-Sossa et al., 2011). El grado de claudicación es comúnmente determinado con base en la clasificación definida por Obel (1948), pero recientemente algunos autores (Meier et al., 2019) han realizado modificaciones a dicha escala y propusieron un método de cinco criterios en tres etapas que emplea una escala de gravedad de 0-12; no obstante, este método es muy limitado dado que muchos de los signos en los que se basan los criterios pueden no estar presentes en la fase preclínica (con la excepción del pulso digital).

En el presente caso clínico, dado que el paciente se mantenía echado y sin ningún intento de levantarse, se asignó un grado 5 , el cual se asigna cuando el animal se niega a moverse a menos que sea forzado; que de acuerdo con la EEAP (2020), es el grado más 
severo. Al respecto, Mitchell et al. (2015) describen que es común que los caballos gravemente afectados tomen una postura específica para cambiar su peso sobre sus patas traseras, debido al intenso dolor que frecuentemente se acompaña de ansiedad y fasciculaciones musculares. Es de vital importancia evitar los periodos prolongados en decúbito de los animales debido a que pueden ocasionar ulceras por presión (Mitchell et al., 2015).

La falta de control adecuado del dolor es una razón común por la cual los caballos afectados por laminitis son finalmente sometidos a la eutanasia (Bamford, 2019). Los fármacos antiinflamatorios no esteroideos (AINE) como flunixina meglumina $(1.1 \mathrm{mg} / \mathrm{kg}$ vía oral (VO) o intravenosa (IV) y fenilbutazona (2-6 mg $/ \mathrm{kg}$ VO), en dosis bajas, se utilizan comúnmente como forma de analgesia y para tratar las respuestas inflamatorias inducidas por laminitis. Para la etapa crónica de laminitis los AINE inhidores de ciclooxigenasa tipo 2 (COX-2), como el firocoxib, que proporcionan un alivio del dolor seguro y eficaz. Los inhibidores no selectivos de la COX pueden ser mejores en las etapas aguda y de desarrollo de la laminitis, ya que la función vascular todavía está presente (Orsini, Wrigley y Riley, 2010). Un vasodilatador como la acepromacina (0.002$0.0066 \mathrm{mg} / \mathrm{kg}$ VO o IV o intramuscular (IM), puede ser de utilidad para contrarrestar la disfunción vascular en la falange (Parks, 2009). La acepromacina aumenta el flujo sanguíneo digital y laminar en caballos normales. Los efectos tranquilizantes de la acepromacina pueden estimular al caballo a acostarse, lo que puede ser beneficioso. Más recientemente, se ha desarrollado el interés en el uso de otros agentes analgésicos como la lidocaína y la ketamina, administrados como una infusión intravenosa, o narcóticos, ya sea como una infusión continua, epidural o intravenosa (Parks, 2009).

Por otro lado, muchos factores deben tenerse en cuenta, especialmente si existe rotación de la tercera falange, suceso que determina un pronóstico reservado en la gran mayoría de los casos (Londoño-Sossa et al., 2011). Algunos autores consideran que un ángulo mayor a 11 es de mal pronóstico para el retorno a la función atlética (Londoño-Sossa et al., 2011). Los estudios radiográficos para evaluar el grado de rotación palmar / plantar de la falange distal que mostro que en la paciente en el presente estudio, dicho ángulo fue de 50, lo que confirma el diagnóstico de laminitis (Sherlock y Parks, 2013). Stick et al. (1982) sugieren que los caballos con una rotación inferior a 5.5ำ tienen un mejor pronóstico para volver a las actividades deportivas que los caballos con una rotación superior a 11.5․ Sin embargo, Paula et al. (2020) reportaron que los caballos que presentaron una rotación de entre $10.5^{\circ}$ y $11.02^{\circ}$, volvieron a sus actividades normales.

El uso de herrajes terapéuticos para tratamiento de laminitis se encuentran el wodem shoe, herraduras de banana con plantillas wedge, herraduras de aluminio con plantillas de banqueta pegadas, representan la primera opción en el tratamiento de laminitis crónica (Estrada, 2011). La tenotomia del tendón flexor digital profundo es una opción más en el tratamiento de caballos laminiticos, ya que ayuda a mejorar la biomecánica, disminuyendo la fuerza de tensión que este tendón ejerce sobre p3, permitiendo la desrotacion, aunque el postoperatorio y la rehabilitación requerirán de medicamentos y tiempo para reestablecer la funcionalidad de la extremidad (Carmona y López, 2011). 
Las radiografías están indicadas en cada caso sospechoso laminítico porque proporcionan información valiosa sobre la presencia, gravedad, cronicidad relativa y naturaleza progresiva de la enfermedad (Sherlock y Parks, 2013). La interpretación del caballo con sospecha de laminitis es más complejo que identificar únicamente la presencia o ausencia de rotación dorsal (Sherlock y Parks, 2013). Una evaluación completa de las radiografías es esencial ya que muchas anormalidades pueden ser evidentes al mismo tiempo (Parks, 2007).

El factor más importante en el pronóstico a largo plazo del caballo con laminitis es la extensión de la patología laminar que influye en el grado de inestabilidad entre la falange distal y pared delcasco Sin embargo, esto es muy difícil de determinar. El diagnóstico avanzado con el uso de imágenes radiológicas junto con los signos clínicos puede ayudar en evaluaciones futura, pero actualmente la evaluación de un caballo con laminitis se basa en prestar mucha atención a los detalles de la serie simple radiografías, evaluación clínica y en ocasiones estudios venográficas.

Afortunadamente, el paciente logró recuperarse satisfactoriamente, situación que representa un caso exitoso, considerando que aproximadamente el $75 \%$ de los caballos tratados por laminitis no retornan a su respectiva actividad física, y en muchos casos son sacrificados en última instancia (Londoño-Sossa et al., 2011).

\section{CONCLUSIÓN}

El tratamiento con antiinflamatorios, herrajes terapeúticos, además del trabajo en equipo "veterinario, herrador, propietario y caballerango" favorecieron el crecimiento y dureza del casco, teniendo éxito en el tratamiento de laminitis.

\section{LITERATURA CITADA}

AMERICAN ASSOCIATION OF EQUINE PRACTITIONERS. 2020. Laminitis prevention and treatment. https://aaep.org/horsehealth/laminitis-prevention-treatment

BAMFORD NJ (2019). Clinical insights: Treatment of laminitis. Equine Veterinary journal. 51:145-146. https://doi.org/10.1111/evj.13055

CARMONA JU, Lopez C. 2011. Tendinopatía del tendón flexor digital superficial y desmopatía del ligamento suspensorio en caballos: fisiopatología y terapias regenerativas. Archivos de Medicina Veterinaria. 43(3): 203-214. http://dx.doi.org/10.4067/S0301-732X2011000300002

ESTRADA UM. 2011. Artículo de revisión fundamentos de podología equina: Recorte balanceado y herraje fisiológico. Revista de Ciencias Veterinarias. 29(2): 41-55. http://www.revistas.una.ac.cr/index.php/veterinaria/index

LONDOÑO-SOSSA J, Robledo-Salgado JC, Cruz-Amaya JM. 2011. Tratamiento quirúrgico de Laminitis crónica: reporte de un caso. Revista Lasallista de Investigación. 8(1): 96-103. http://www.scielo.org.co/pdf/rlsi/v8n1/v8n1a11.pdf 
MEIER A, de Laat M, Pollitt C, Walsh D, McGree J, Reiche DB, Salis-Soglio MV, WellsSmith L, Mengeler U, Mesa SD, Droegemueller S, Sillence MN. 2019. A "modified Obel" method for the severity scoring of (endocrinopathic) equine laminitis. Peer J. 7:e7084. https://dx.doi.org/10.7717\%2Fpeerj.7084

MITCHELL CF, Fugler LA, Eades SC. 2015. The management of equine acute laminitis. Veterinary Medicine: Research and Reports. 6: 39-47. http://dx.doi.org/10.2147/VMRR.S39967

OBEL N. 1948. Studies on the histopathology of acute laminitis. D. Phil., Thesis. Almquisst and Wiksells Boktryckeri, Uppsala. https://www.cabdirect.org/cabdirect/abstract/19522200184

PARKS A. 2007. Patterns of displacement of the distal phalanx and its sequelae. In: Proceedings of 46th British Equine Veterinary Association Congress, Equine Veterinary Journal, Newmarket. Pp. 204-205.. https://www.semanticscholar.org/paper/Proceedingsof-the-49th-British-Equine-Veterinary-

PARKS A. 2009. Acute and Chronic Laminitis - An Overview. In: 2009 Proceedings of the American Association of Equine Practitioners - Focus Meeting Focus on the Foot, Columbus, Ohio, USA [online] 2009; 132-139. https://www.ivis.org/library/aaep/aaepfocus-meeting-focus-on-foot-columbus-2009/acute-and-chronic-laminitis-an-overview

PAULA LAO, Lera KRJL, Schuh BRF, Silva FFA, Michelon do Nascimento E, Pagliosa GM. 2020. Laminite endocrinopática em equinos com síndrome metabólica: características clínicas, tratamento e evolução em três pacientes - relato de caso. Arquivo Brasileiro de Medicina Veterinária e Zootecnia. 72(4): 1375-1380. https://doi.org/10.1590/1678-4162-11778

SHERLOCK C, Parks A. 2013. Radiographic and radiological assessment of laminitis. Equine Veterinary Education. 25(10): 524-535. https://doi.org/10.1111/eve.12065

STICK JA, Jann HW, Scott EA, Robinson NE. 1982. Pedal bone rotation as an indicator of laminitis in horses. Journal of the American. Veterinary Medical Association. 180: 251-253. https://www.ncbi.nlm.nih.gov/pubmed/7056672 